\title{
End-to-End 5G Service Deployment and Orchestration in Optical Networks with QoE guarantees
}

\author{
Rafael Montero, Fernando Agraz, Albert Pagès, Salvatore Spadaro \\ Universitat Politècnica de Catalunya, Jordi Girona 31, 08034 Barcelona, Spain \\ e-mail:spadaro@tsc.upc.edu
}

\begin{abstract}
The upcoming $5 \mathrm{G}$ deployments will impose stringent requirements. Optical networks control and resource orchestration is set to potentially turn into software-defined approaches in order to address such requirements. As a result, there rises a need for an architectural scheme capable of supporting the different types of services defined for $5 \mathrm{G}$ verticals.

We present in this paper an architecture enabling end-to-end (E2E) provisioning and monitoring of such 5G services over optical network segments. In particular, the scenario considers the coordination of various optical enabled network segments by a higher level E2E Orchestrator, which provides of network slice deployment and is able to guarantee agreed levels of Quality of Experience (QoE). Moreover, we discuss an example of 5G service provisioning using the proposed architecture to demonstrate its behaviour in front of different network events.
\end{abstract}

Keywords: Orchestration, Optical Networks, Network Slicing, 5G Service Deployment, QoE Monitoring.

\section{INTRODUCTION}

Under the keyword 5G, the future network infrastructure aims at the removal of limitations to current networks by addressing the challenges in managing, controlling and orchestrating a fully softwarized network and services running on the (multi-domain) infrastructure. In this $5 \mathrm{G}$ era, network infrastructure will go well beyond the evolution of today's transport networks, and have to be properly designed to support end-to-end 5G-enabled vertical applications [1]. In this direction, $5 \mathrm{G}$ promises to deliver not only a better performing network, but also to become an infrastructure capable of supporting ubiquitous services, while at the same time meeting the performance and commercial requirements of multiple stakeholders.

5G networks look forward to support demanding next-generation services. In this matter, enhanced mobile broadband (eMBB), ultra-reliable and low latency communications (URLLC) and massive machine-type communications (mMTC) are the $5 \mathrm{G}$ use cases classes defined by $3 \mathrm{GPP}$. Different $5 \mathrm{G}$ vertical industries such as automotive, manufacturing, energy, eHealth, entertainment, among others, are set to make use of these types of services [1].

It is therefore required to provision an infrastructure that meets the requirements of verticals and utilises the mobile and fixed infrastructure. 5G has to have the ability to hide from the end users, enterprises and vertical businesses the complexity and heterogeneity in terms of technology and administrative domains of the physical and virtual infrastructures over which the tailored communication systems are deployed and operated. In such context, 5G network providers offer networks as a service to create "network slices" to be flexibly and efficiently allocated to use cases, by provisioning logically isolated end-to-end networks. Network Slicing is seen as the most promising enabler in the remarkable paradigm shift from $4 \mathrm{G}$ to NFV/SDN-enabled 5G era; software-Defined Networking (SDN) and Network Function Virtualisation (NFV) will be key elements for the $5 \mathrm{G}$ deployments. The introduction of network slicing in $5 \mathrm{G}$ pursues to achieve the sharing of both network and data centres resources, thereby substantially reducing the capital expenditure for $5 \mathrm{G}$ network operators.

A Network Slice Instance (NSI) comprises a set of Network Functions (NFs), and resources to run these NFs. An NSI can include more Network Slice Sub-network Instances (NSSIs).

A number of Key Performance Indicators (KPI) is being defined to evaluate the service experience of the customer. In this regard, the Quality of Experience (QoE) concept arises as one of the main leading aspect to be optimized. However, ensuring and managing proper levels of QoE is a very challenging task, which derives also from established Quality of Service (QoS) specifications and Service Level Agreements (SLA). To this end, special focus on the management and control aspects of the provisioned network slices has to be put, while performing guaranteed an optimized slice deployment and lifecycle, not only from the physical infrastructure perspective, but also from the user and/or vertical business side.

In support of 5G network slicing, a functional architecture to allow the provisioning of network slices has to be designed. In this regard, the architecture has to include a control layer, for network configuration and slicing, and an orchestration layer for e2e and cross-segment slice provisioning optimization and coordination.

In this paper, we detail a potential architecture to be used to support network slices provisioning with the proper level of QoE guarantees. 


\section{E2E ORCHESTRATION ARCHITECTURE}

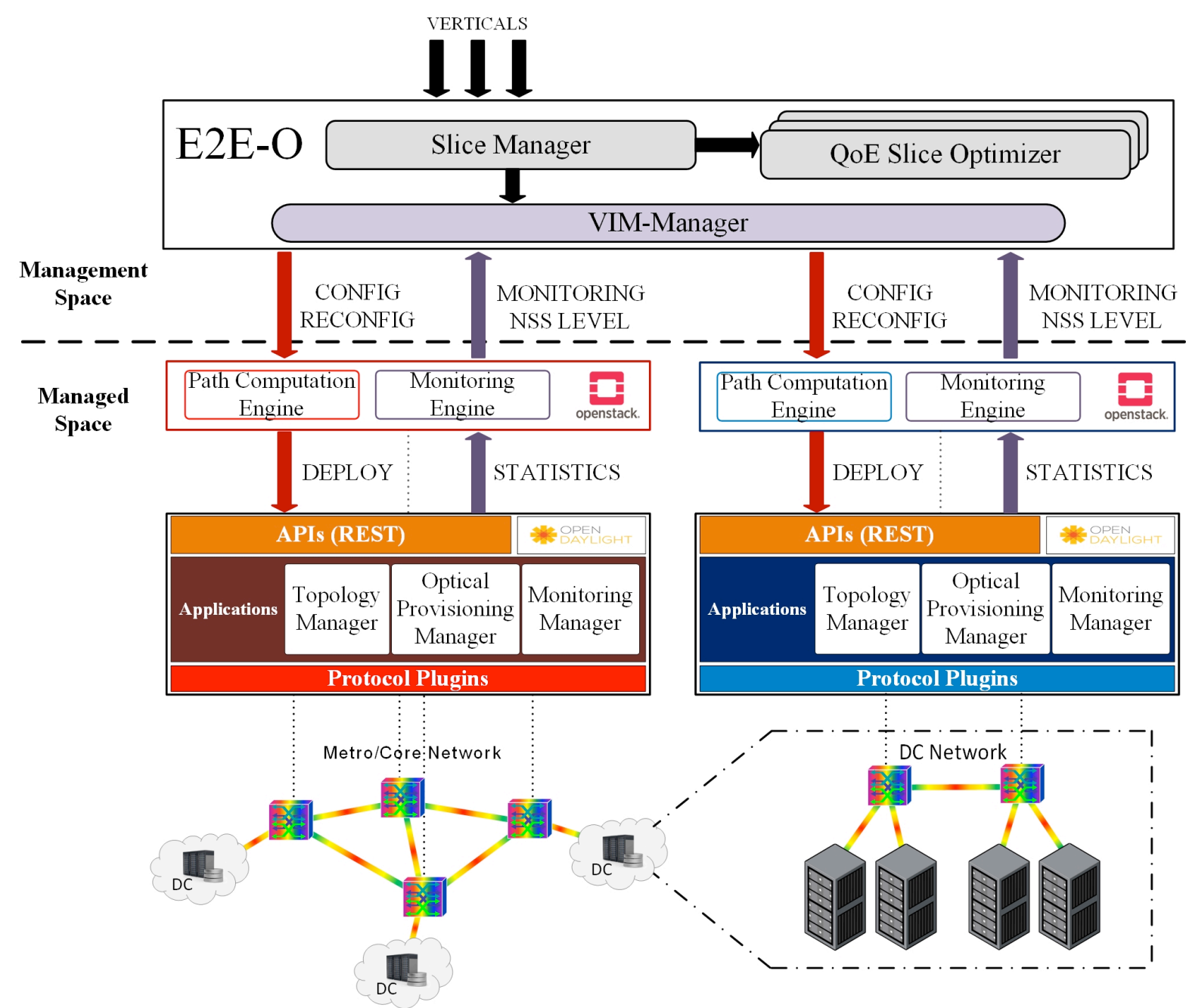

Figure 1. E2E Orchestration Architecture.

Figure 1 depicts the architecture proposed to provide E2E network slices' infrastructure in support of 5G services. Such architecture is split in two logical spaces, the Management Space (top) and the Managed Space (bottom). The Management Space comprises the entities aimed to provide E2E network slices control and management, and the Managed Space refers to the infrastructure segments (Metro/Core and DC, in our case) that are going to be used to provide the network sub-slices to compose such E2E network slices. As illustrated in the figure, the segments in the managed space have their own control and management systems, which are the entry points for the upper space management and orchestration agents. The following subsections describe more in detail the elements and functionalities of each space.

\subsection{Management Space}

At the top level, the E2E Orchestrator (E2E-O) is the responsible for managing the E2E network slice creation. To do this, it provides a communication interface to the verticals so they can request network slices to support different types of $5 \mathrm{G}$ services (e.g. eMBB, URLLC, mMTC).

In particular, the Slice Manager is the component that centralizes the E2E network slice creation. It computes the high-level deployment of the slice (e.g., segments to pass through, dimensioning the number of resources needed, etc.) and contacts the VIM Manager in order to demand the required network connectivity and the allocation of IT resources for the proper deployment of the slice. The Slice Manager also provides the QoE Optimizer with the KPIs specification for type of service to be supported. The QoE Slice Optimizer uses cognition-based mechanisms to guarantee that the QoS/QoE requested for the slice is maintained during operation. To do this, an instance of the QoE Optimizer is created per slice.

Upon the request from the Slice Manager, the VIM Manager coordinates with the VIMs controlling each network segment (i.e., Metro/Core Network and DC network) to either configure or reconfigure network and/or IT requirements. While the configuration is triggered by the Slice Manager at slice provisioning time, the reconfiguration is initiated by the QoE Optimizer upon the potential degradation of service QoE agreements 
during the slice operation. To predict such situations, the QoE Optimizer collects the aggregated network subslice monitoring information from each underlying segment (through the VIM) to further analyse it using predictive machine-learning algorithms. Note that, as depicted in Fig. 1, the VIM of each segment is composed of the orchestrator and the SDN controller.

\subsection{Managed Space}

The Managed Space is composed of the infrastructures of the segments that are managed by the E2E-O. Such segment infrastructures consist of orchestration (top), control (middle) and physical resources (bottom). The segments are independent from each other in terms of components and resources. For example, in our case, the Metro/Core segment consists only of network resources while the DC Network segment contains both network and IT resources. Furthermore, each segment is provided with its own SDN controller and orchestrator.

The Metro/Core segment is aimed to provide network connectivity between the other segments of E2E scenario. To do this, the Path Computation Engine (PCE) of the Openstack-based orchestrator computes the lightpaths to be configured to provide connectivity also satisfying the QoS/QoE requirements requested by the E2E-O. Such computation is sent to the Optical Provisioning Manager (OPM) module of the Opendaylightbased SDN controller, which is responsible for the configuration of the optical data plane devices. An extended version of the OpenFlow protocol is used to this end [2].

The DC segment provides the virtualized computation infrastructure (i.e., VMs plus network connectivity) to host the Virtual Network Functions (VNFs) that will support the service requested by the vertical. Hence, upon the request coming from the E2E-O, the PCE of the orchestrator of the DC segment computes the virtual data center infrastructure to be deployed taking into account the requirements of the requested service. Note that, in this case, the PCE is enhanced with algorithms that allow optimized IT plus network deployment computation. Then, the Openstack-based orchestrator deploys the VMs as established by the PCE and contacts the Opendaylight SDN controller to configure the network connectivity between them. As in the Metro/Core segment case, the optical DCN is configured by means of the extended OpenFlow protocol.

To enable the QoE cognitive management at the E2E-O, both Metro/Core and DC segments are equipped with a QoS/QoE monitoring stack. In particular, both SDN controllers contain a Monitoring Module (MM) that collects statistical information from the data plane. Such per-device monitoring information is aggregated by network sub-slice at the Monitoring Engine (ME) of the segment orchestrator, and sent to the E2E-O, which uses it to guarantee the compliance of the requested QoS/QoE requirements.

\section{TECHNICAL USE CASES AND WORKFLOWS}

As said before, the scope of the presented architecture is the provisioning of network slices with QoE guarantees in support of $5 \mathrm{G}$ services. In this regard, we define two main technical use cases (TUCs) to be materialized through the proposed architecture, namely, slice provisioning and runtime maintenance of QoE metrics of services deployed over the slice. While the first TUC relates to more traditional infrastructure configuration to support services on top, with the addition of QoE-awareness, the second TUC falls under the category of Self Optimized Networks (SONs), as defined in [3], which is highlighted as a crucial TUC for network provisioning architectures in the context of $3 \mathrm{G}$ systems and future wireless and telecom systems. Indeed, architectures providing the capacity of self-learning and automated optimization of resources to cope with dynamic conditions that may affect the quality of the deployed virtual infrastructure are a must in 5G networks, hence our focus on providing the architecture and the steps for its realization. Next, we summarize the steps for both considered TUCs. Figure 2 depicts a high level workflow highlighting the main involved entities for slice provisioning (left) and runtime QoE maintenance/optimization (right).

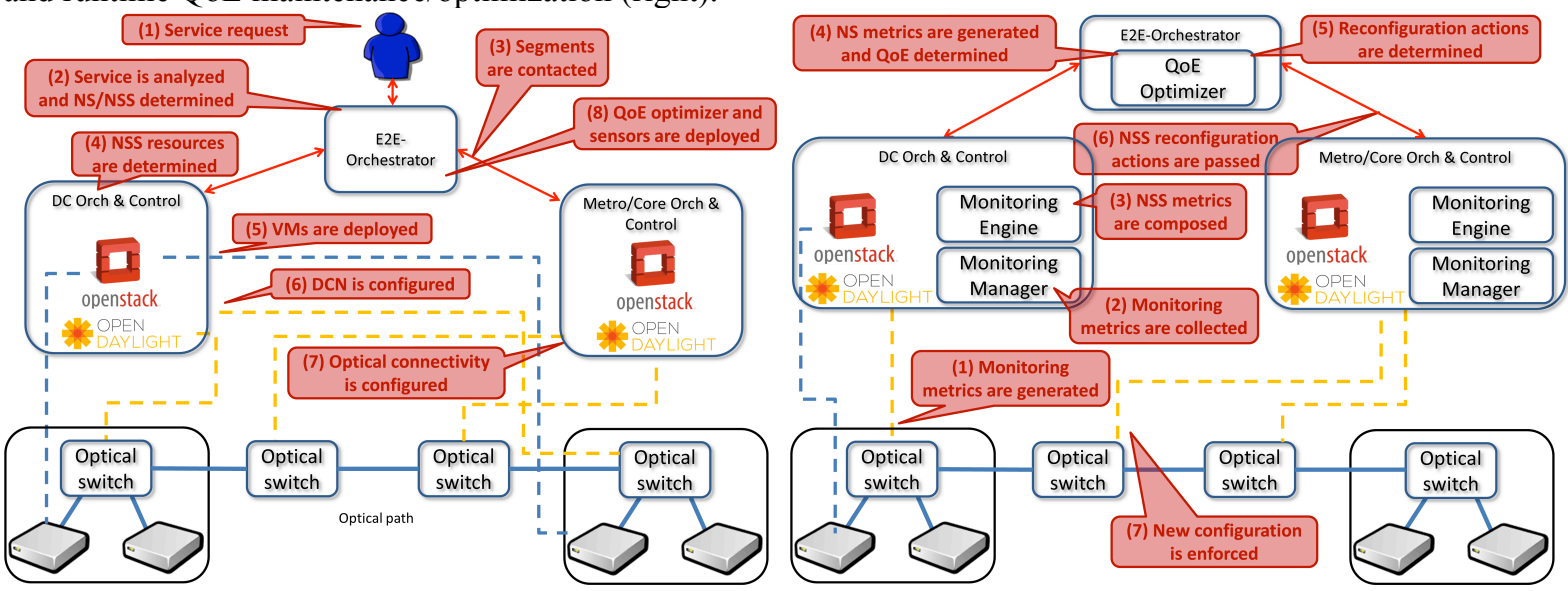

Figure 2. Workflows for network slice provisioning (left) and runtime QoE optimization of slices (right). 


\subsection{Slice provisioning with QoE guarantees}

The process begins when the vertical demands for the required network service (Step 1). Upon such request, at the E2E-O, the Slice Manager analyses the specific requirements for the particular service, that is, which is the needed virtual infrastructure to be set-up (Step 2). This translates into computational resources (e.g., VMs) and network connectivity between configured computational resources. To decouple the specific technological details of the configuration from the service/slice orchestration, the abovementioned VIM-M is introduced. The VIM-M takes the outputs of the Slice Manager, which already has split the resulting NS into the necessary NSS, and contacts the corresponding segment to set-up the different NSSs to compose the E2E virtual infrastructure (Step 3). At the northbound of each segment, the segment orchestrator analyses the resources needed for each NSS and coordinates the underlying resource configuration (Step 4). On the one hand, to deploy the necessary computational resources, the DC orchestrator creates the VM instances at particular servers, while also configuring the IP network to allow for exchange of data between VMs (Step 5). To set up the necessary connectivity at the data plane, the corresponding SDN controller configures the optical DCN (Step 6). In parallel, to interconnect the distributed computational resources across DC sites, the metro/core orchestrator and controller set-up the necessary IP network as well as E2E optical connectivity across the optical metro/core network (Steps 7). In this way, all the NSSs are interconnected and the full NS is set-up.

Lastly, besides the pure NS configuration, to provide QoE-awareness, the Slice Manager configures an instance of the QoE optimizer, particularized for the concrete service at hand as well as the deployment of software sensors that process and aggregate information at the infrastructure level to derive QoE metrics (Step 8). This whole framework will be later on employed to monitor the performance of the deployed NS.

\subsection{Runtime QoE optimization}

After NS resources are deployed and services are running on top, the whole QoE monitoring and optimization starts. First, performance metrics (e.g., bandwidth, latency, packet losses, CPU utilization) are collected at the infrastructure level for each segment (Step 1). The information is collected through the MM at the SDN controllers (Step 2). Such information is then processed by the ME, at the segment orchestration layer, to compose aggregated NSS monitoring information (Step 3). In this regard, several software sensors are employed, which filter the most relevant Quality of Service (QoS) metrics that may effect on the QoE of the service running over the E2E NS. These sensors, named QoE sensors are deployed at the provisioning phase and serve the purpose to particularize the whole monitoring framework to the NS deployed. The generated NSS monitoring information is then collected by the QoE optimizer and aggregated to compose the E2E NS KPIs. Then, such metrics are analysed by a machine learning model which translates the QoS metrics of the slice into QoE metrics, which are the ones relevant for the service running on top (Step 4). Given the output of these metrics, the QoE optimizer determines if the QoE is satisfied or not. In the latter case, and given the received monitoring information, the QoE optimizer determines corrective actions to be carried out, that is, necessary virtual infrastructure reconfigurations to meet again the desired QoE levels (Step 5). These actions could be the result of different physical infrastructure events, for example the bandwidth saturation on fibre links or insufficient CPU capacity at VMs, which then require the most suitable reconfiguration to be compensated, e.g. increase of the bandwidth of an optical connection or scaling up the resources of a VM. Such actions are then passed down to the corresponding Orchestration and Control layers of the different segments (Step 6) and enforced onto the underlying physical infrastructure (Step 7).

\section{CONCLUSIONS}

An end-to-end architecture enabling $5 \mathrm{G}$ network slices has been introduced. The presented architecture focuses on QoE-awareness virtual infrastructure provisioning and runtime optimization of the deployed resources to maximize service quality over the time. In this regard, potential use cases and corresponding workflows have been discussed to exemplify how the proposed architecture provides capabilities for QoE guarantees, addressing future needs for optical networks and DC sites upon requirements imposed by upcoming $5 \mathrm{G}$ infrastructures.

\section{ACKNOWLEDGEMENTS}

This work has been supported by the H2020 5GPPP SLICENET project (H2020-ICT-2016-2/761913) and the Spanish Government through project ALLIANCE (TEC2017-90034-C2-2-R) with FEDER contribution.

\section{REFERENCES}

[1] 5G-PPP 5G Architecture White Paper, Version 2.0, December 2017.

[2] S. Spadaro, et al., "Resource Orchestration in SDN-based Future Optical Data Centres", 20th International Conference on Optical Network Design and Modeling (ONDM 2016), Cartagena (Spain), May 2016.

[3] 3GPP TR 28.801, Study on management and orchestration of network slicing for next generation network, September 2017. 